\section{Serratia marcescens infection or hypoxic-ischaemic encephalopathy in neonates: Is magnetic resonance imaging a problem-solving tool?}

To the Editor: We read with great interest the retrospective case series by A Madide and J Smith, ${ }^{[1]}$ describing brain ultrasound (US) findings of neonates with Serratia marcescens hospital-acquired infections. S. marcescens is a Gram-negative organism that frequently colonises neonatal intensive care units (NICUs). This bacterium can cause severe brain infections in neonates, with irreversible neurological damage and long-term neurodevelopmental impairment. The authors conclude that in babies with acute onset of the illness, US scans allow doctors to highlight pathological changes in the brain immediately before the microbiological diagnosis of infection, and to follow the evolution of those lesions. We are in complete agreement with their comments regarding the importance of accurate and timely diagnosis: early treatment, to minimise the bacterial load, seems to have a great impact on outcome. Moreover, it is important to underline that transfontanellar US scans remain the first-line modality of imaging because of their safety, low cost and especially because of their wide availability. ${ }^{[2]}$

We would like to share our experience with a similar case, in a child with a prenatal diagnosis of partial agenesis of the corpus callosum and multiple intestinal atresia, born at 37 weeks' gestational age, who developed postnatal hypoxic-ischaemic encephalopathy (HIE). The child underwent more than one surgical procedure to correct bowel atresia during the first week of life. During his stay in the NICU, he developed seizures and lethargy. In light of suspected infection, the neonatologist performed microbiological cultures and started broad-spectrum antibiotics. Meanwhile, the child underwent a transfontanellar US scan that showed increased echogenicity of the subcortical white matter and extensive cystic periventricular leukomalacia, well-known findings interpreted as sequelae of HIE.

Magnetic resonance imaging (MRI) of the brain confirmed severe periventricular cystic changes around the bodies of the lateral ventricles, and highlighted the presence of two focal lesions in the occipital lobes, with a hypointense centre on T1 weighted and a hyperintense on T2 weighted, and fluid attenuation inversion recovery (FLAIR) images with a rim, mildly hyperintense on $\mathrm{T} 1$ and hypointense on $\mathrm{T} 2$. The central core of the masses restricts strongly on the diffusion weighted imaging (DWI) and apparent diffusion coefficient (ADC) map. MRI features are consistent with cerebral abscesses (Fig. 1).

Neurosurgery and abscess drainage were immediately performed and the culture of the abscess fluid revealed S. marcescens.

Confirmation of the brain US finding by MRI evaluation is very helpful in guiding therapy, especially when US scans usually do not allow a perfect distinction between the site of a previous haemorrhage and an abscess. ${ }^{[3]}$

MRI has been gaining increasing importance in the evaluation of brain infections and abscesses because of its high sensitivity and specificity. A major disadvantage is that MRI requires the transport and possibly long sedation of critically ill patients, who are often haemodynamically unstable.

\section{Narese}

Department of Radiology, Department of Pathobiology and Medical Biotechnology (DIBIMED), University of Palermo, Palermo, Italy; Neuroradiology Unit, Imaging Department, Bambino Gesù Children's Hospital, Rome, Italy

donatella.narese@virgilio.it

\section{Auriti}

Neonatal Intensive Care Unit, Department of Neonatology, Bambino Gesù Children's Hospital, Rome, Italy

\section{Longo}

Neuroradiology Unit, Imaging Department, Bambino Gesù Children’s Hospital, Rome, Italy

1. Madide A, Smith J. Intracranial complications of Serratia marcescens infection in neonates. S Afr Med J 2016;106(4):36-38. https://doi.org/10.7196/SAMJ.2016.v106i4.10206

Messerschmidt A, Prayer D, Olischar M, et al. Brain abscesses after Serratia marcescens infection on a neonatal intensive care unit: Differences on serial imaging. Neuroradiology 2004;46(2):148-152. https://doi.org/10.1007/s00234-003-1140-8

3. Hirooka TM, Fontes RB, Diniz EM, et al. Cerebral abscess caused by Serratia marcescens in premature neonate. Arq Neuropsiquiatr 2007;65(4A):1018-1021. https://doi.org/10.1590/S0004$282 \times 2007000600021$
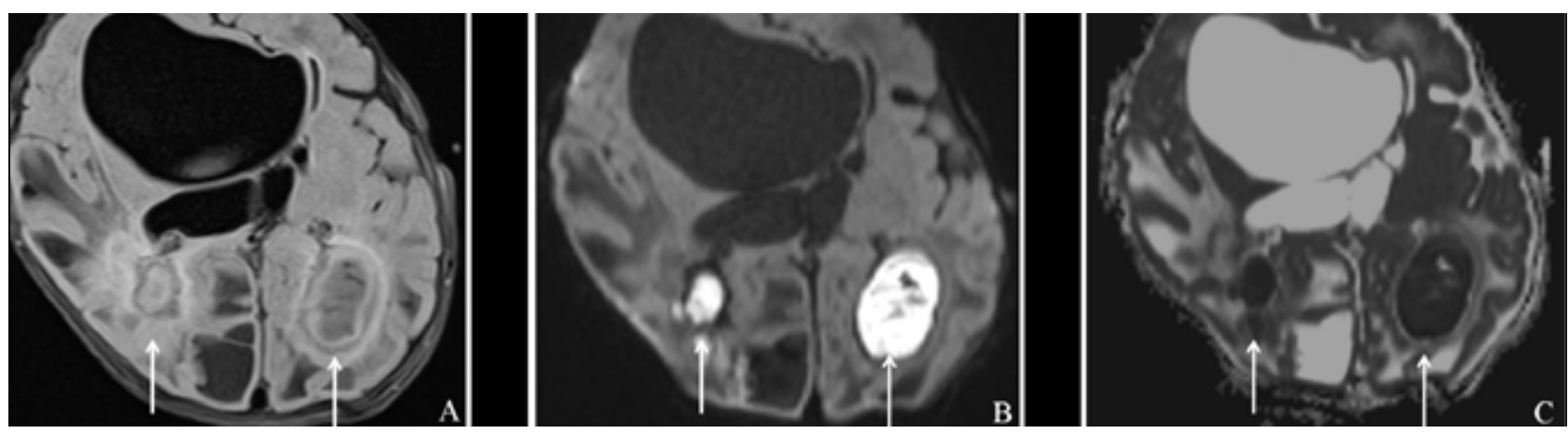

Fig. 1. (A) Axial FLAIR images show two bilateral focal lesions (arrows) in the occipital lobes with a hyperintense centre and with a mildly hypointense rim. A significant amount of FLAIR hyperintense signal abnormality surrounds the lesions. The lesion's content shows diffusion restriction, being bright signal in $D W I(B)$ and low signal in $A D C(C)$. 